SLAC-PUB-12925

October 2007

\title{
Performance study of $\mathrm{Si} / \mathrm{CdTe}$ semiconductor Compton telescopes with Monte Carlo simulation
}

\author{
Hirokazu Odaka $^{a, b, *}$, Shin'ichiro Takeda ${ }^{a, b}$, Shin Watanabe ${ }^{a}$, Shin-nosuke Ishikawa ${ }^{a, b}$, \\ Masayoshi Ushio $^{\mathrm{a}, \mathrm{b}}$, Takaaki Tanaka ${ }^{\mathrm{a}, \mathrm{b}}$, Kazuhiro Nakazawa ${ }^{\mathrm{a}}$, Tadayuki Takahashi ${ }^{\mathrm{a}, \mathrm{b}}$, \\ Hiroyasu Tajima ${ }^{\mathrm{c}}$, Yasushi Fukazawa ${ }^{\mathrm{d}}$ \\ a Department of High Energy Astrophysics, Institute of Space and Astronautical Science (ISAS), Japan Aerospace Exploration Agency (JAXA), \\ 3-1-1 Yoshinodai, Sagamihara 229-8510, Japan \\ ${ }^{\mathrm{b}}$ Department of Physics, Graduate School of Science, University of Tokyo, Hongo 7-3-1, Bunkyo 113-0033, Japan \\ ${ }^{\mathrm{c}}$ Stanford Linear Accelerator Center (SLAC), 2575 Sand Hill Road, Menlo Park, CA 94025, USA \\ ${ }^{\mathrm{d}}$ Department of Physical Science, Hiroshima University, 1-3-1 Kagamiyama, Higashi-Hiroshima 739-8526, Japan
}

\begin{abstract}
A Compton telescope with high angular resolution and high energy resolution is a promising detector for the next generation of astrophysics space missions aiming at hard $\mathrm{X}$-rays and sub-MeV/MeV gamma-rays. We have been working on a semiconductor Compton camera based on silicon and cadmium telluride ( $\mathrm{Si} / \mathrm{CdTe}$ Compton telescope). The soft gamma-ray detector (SGD) employs a $\mathrm{Si} / \mathrm{CdTe}$ Compton camera combined with a well-type active shield. It will be mounted on the NeXT mission, proposed to be launched around 2012. One Compton camera module in the SGD will consist of 24 layers of double-sided silicon strip detectors and four layers of CdTe pixel detectors. We carried out Monte Carlo simulations to investigate the basic performance of the detector. Design parameters of devices required in the simulation, such as energy resolution and position resolution of the detector, are based on the results from our prototype detector. From the simulation using current design parameters, the detection efficiency is found to be higher than $10 \%$ at $\sim 100 \mathrm{keV}$ and the angular resolution to be $9^{\circ}$ and $4.4^{\circ}$ at $120 \mathrm{keV}$ and $330 \mathrm{keV}$, respectively. The effects of changing the design parameters are also discussed.
\end{abstract}

\section{Introduction}

Gamma-rays in the energy range from several tens of $\mathrm{keV}$ to several $\mathrm{MeV}$ provide an important window to the study of energetic phenomena in the universe such as nucleosynthesis and particle acceleration. These phenomena are observed in objects such as pulsars, stellar blackhole candidates, supernova remnants, active galactic

\footnotetext{
${ }^{*}$ Corresponding author. Department of High Energy Astrophysics, Institute of Space and Astronautical Science (ISAS), Japan Aerospace Exploration Agency (JAXA), 3-1-1 Yoshinodai, Sagamihara 229-8510, Japan.

E-mail address: odaka@astro.isas.jaxa.jp (H. Odaka).
}

nuclei, and gamma-ray bursts. The observational sensitivity in this energy band, however, is relatively low due to high background levels, low detection efficiency, and limited angular resolution. Compton telescopes are promising detectors to overcome these problems since the direction of incident $\gamma$-rays is constrained by Compton kinematics, greatly reducing the background as compared with detectors which employ a coded mask or a collimator.

The first successful Compton telescope in orbit was COMPTEL aboard the Compton gamma-ray observatory (CGRO) [1]. COMPTEL observations provided pioneering results including all sky imaging from 1 to $30 \mathrm{MeV}$ and spectroscopy of $\mathrm{MeV}$ gamma-ray lines [2]. But the number of detected objects was very small, with only 32 sources 
detected [3]. Thus improving the sensitivity is the key goal of the next generation detectors. This requires a higher detection efficiency, a lower instrumental background and better angular resolution [4]. A high density detector array with improved energy and position resolution is needed. With this point of view, Compton telescopes based on position-sensitive semiconductor detectors, such as $\mathrm{Si}, \mathrm{Ge}$, CZT, and CdTe, have been proposed and developed by various groups [5-10].

Our group has proposed building a $\mathrm{Si} / \mathrm{CdTe}$ Compton telescope based on the recent achievements of the development of $\mathrm{Si}$ and $\mathrm{CdTe}$ semiconductor imaging detectors with high energy resolution [9]. A schematic drawing of a $\mathrm{Si} / \mathrm{CdTe}$ Compton telescope is shown in Fig. 1. The telescope is based on a hybrid semiconductor gamma-ray detector consisting of layers of thin $\mathrm{Si}$ and CdTe to detect photons in a wide energy band $(0.05-1 \mathrm{MeV})$. Through using a prototype Compton camera, we succeeded in reconstructing images and spectra of gamma-rays from 81 to $662 \mathrm{keV}$. We were also able to make photon polarization measurements [11-13].

The $\mathrm{Si} / \mathrm{CdTe}$ Compton telescope is adopted as one of instruments on board the NeXT (New X-ray Telescope/ Non-thermal Energy eXploration Telescope) mission, proposed in Japan as a successor to the current Suzaku X-ray mission. A detailed description of the instrument, refered to as the SGD (soft gamma-ray detector), is presented in Takahashi et al. [14]. Since the SGD must outperform previous soft $\gamma$-ray instruments in background rejection capability, the optimization of the design is of great importance. For this purpose, we have studied the performance of the $\mathrm{Si} / \mathrm{CdTe}$ Compton telescope by using Monte Carlo simulations. Experimental results of the prototype Compton camera are used for assuming parameters of devices, for example, energy and position resolution of the detector. Initial results of simulations regarding the detection efficiency and performance as a polarimeter were presented in our previous publication [15]. In this paper, we present new results on the performance of the $\mathrm{Si} / \mathrm{CdTe}$ detector in the SGD. In

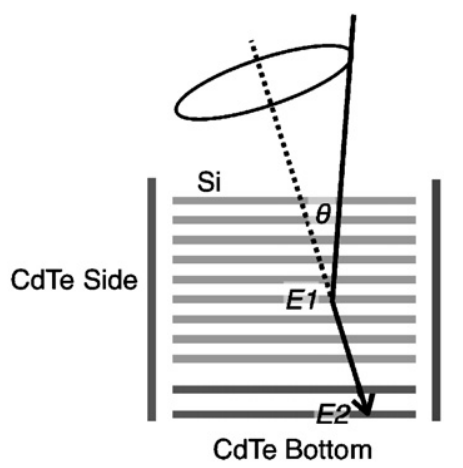

Fig. 1. Schematic picture of a $\mathrm{Si} / \mathrm{CdTe}$ Compton camera. An incident photon is scattered at a silicon detector and then absorbed at a $\mathrm{CdTe}$ detector. $E_{1}$ and $E_{2}$ are energy deposited of the two hits, and $\theta$ is the scatter angle. particular, the detection efficiency as a function of various design parameters and the angular resolution as a function of various data selection are described in detail.

\section{Simulation Setup}

Fig. 2 shows a schematic diagram of the geometry of the detector used in the simulation. The telescope consists of 24 layers of double-sided silicon strip detectors (DSSDs) and four layers of thin CdTe pixellated detectors (CdTe Bottom) with a thickness of $0.5 \mathrm{~mm}$. The sides are also surrounded by CdTe pixel detectors (CdTe Side). In order to lower the background dramatically and thus to improve the sensitivity, we combine a stack of Si strip detectors and CdTe pixel detectors to form a Compton telescope. This configuration is suitable for a Compton camera, since $\mathrm{Si}$ has a small cross-section for photo absorption even for very soft $\gamma$-rays (e.g. $\sim 80 \mathrm{keV}$ ) and CdTe has a large crosssection for photo absorption due to their large atomic numbers $\left(Z_{\mathrm{Cd}}=48, Z_{\mathrm{Te}}=52\right)$. The telescope is then mounted inside the bottom of a well-type active shield to further reduce the background by adopting a new concept, narrow-Field-of-View (FOV) Compton telescope $[14,16]$.

The size of an individual DSSD is $50 \mathrm{~mm} \times 50 \mathrm{~mm}$ with a thickness of $0.5 \mathrm{~mm}$, and each DSSD has 125 strips on each side with a strip pitch of $0.4 \mathrm{~mm}$. The energy

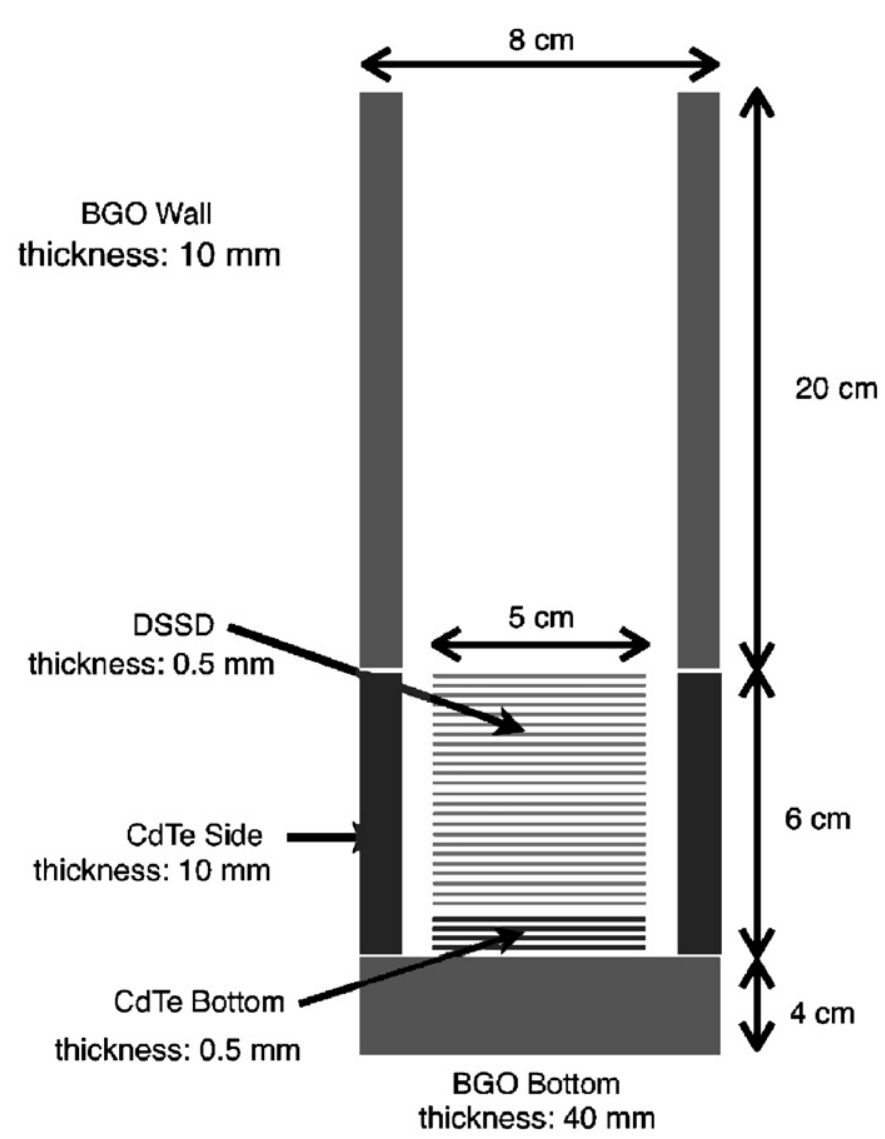

Fig. 2. The simulated geometry of the $\mathrm{Si} / \mathrm{CdTe}$ Compton telescope. 
resolution is assumed to be $1.5 \mathrm{keV}$ (full-width at half-maximum, FWHM), as demonstrated in our previous prototype (also see Ref. [17]). Four layers of CdTe pixel detectors (CdTe Bottom) are also formed as another stack and are placed under the stacked DSSDs. Each CdTe detector consists of $25 \times 25$ pixels and has a size of $50 \mathrm{~mm}$ $\times 50 \mathrm{~mm}$ with a thickness of $0.5 \mathrm{~mm}$. The pixel size is $2 \mathrm{~mm}$ $\times 2 \mathrm{~mm}$. In the simulation, each stack has a frame made of silicon simulating the substrate or electronic devices for data readout, which acts as blocking material around the detector. The thickness is $0.5 \mathrm{~mm}$ in the default setting. In addition to these, four CdTe detectors (CdTe Side) cover individual sides of the DSSDs. The thickness of the material associated with the CdTe Side is $10 \mathrm{~mm}$. In the default setting, only the inner $0.5 \mathrm{~mm}$ of the CdTe Side is activated as a detector and the rest is used as an anticoincidence shield. The energy resolution of the $\mathrm{CdTe}$ detectors are set at $1.5 \mathrm{keV}$ (FWHM). BGO crystals are placed at both the top and the bottom of the Compton camera. The well-type shield is formed by these crystals along with the outer parts of the $4 \mathrm{CdTe}$ Side detectors. The thickness of the BGO is $10 \mathrm{~mm}$ on the side and $40 \mathrm{~mm}$ at the bottom.

In the simulation, incident photons have a power-law spectrum with a photon index of -2.1 ranging from 50 to $400 \mathrm{keV}$, and are generated at the top of the detector, irradiating it uniformly, thus emulating signals from a celestial source. We used the Geant4 simulation toolkit [18] to carry out the simulations and included the G4LECS extension of Kippen $[19,20]$ in order to estimate the effect of Doppler broadening. This broadening is due to the finite momentum of bound electrons in an atom and it results in a deterioration of the angular resolution of Compton telescopes [21].

\section{Compton reconstruction}

The data taken from the $\mathrm{Si} / \mathrm{CdTe}$ Compton telescope allow for two analysis modes. The first is the photo absorption mode and the second is the Compton mode. In the photo absorption mode, the energy deposited in all layers is summed if the corresponding deposited energy exceeds a threshold energy of the detector.

In the Compton mode, events satisfying a condition that a photon is scattered once and then absorbed are selected. Once the locations and energies of the two interactions are measured, the Compton equation allows the calculation of the energy and the direction (as a cone in the sky) of the incident $\gamma$-ray:

$E_{\text {in }}=E_{1}+E_{2}$

$\cos \theta=1-\frac{m_{\mathrm{e}} c^{2}}{E_{2}}+\frac{m_{\mathrm{e}} c^{2}}{E_{1}+E_{2}}$

where $E_{1}$ is the energy deposited by the Compton scattering and $E_{2}$ is that deposited by the photo absorption, $m_{\mathrm{e}}$ is the electron mass and $c$ is the speed of light.
An outline of our Compton reconstruction algorithm is as follows:

(1) Event trigger: The target of analysis is events that have at least one hit which has an energy deposit exceeding the trigger energy of $10 \mathrm{keV}$. Within the event, all hits that deposited energy exceeding $3 \mathrm{keV}$ are identified and collected.

(2) Clustering: Two hits detected at adjacent pixels or strips are combined into one. The energy deposited is the sum of the two and the newly calculated position is the energy-weighted average of the two hit positions. By this operation, an event such that charge is shared between two adjacent pixels or strips is regarded as one hit.

(3) Anti-coincidence: Events which have at least one hit in the active shield (BGO scintillator and a part of the CdTe Side detector) are rejected. The threshold energy is set at $30 \mathrm{keV}$.

(4) Two-hit selection: After these screenings, events which have two hits are selected.

(5) "Real Compton" selection: The energy of the recoil electron of Compton scattering $E_{1}$ is calculated from the other energy deposited $E_{2}$ (assumed to be a photo absorption hit) and the measured scatter angle $\theta$. The value of $\theta$ is determined geometrically from the positions of the two hits and the incident direction of the photon, i.e. the FOV of the well-type shield. The significance of the difference between the measured and calculated $E_{1}$ is estimated taking account the position resolution, energy resolution, and the effect of the Doppler broadening. Finally, events satisfying the criterion that the calculated $E_{1}$ be equal to the measured $E_{1}$ within a certain error $(4 \sigma)$ are selected.

It is worth discussing the physics of the identification of the interactions. If a hit takes place at the CdTe Side detectors, we can clearly distinguish between photo absorption and Compton scattering via geometrical considerations. For other hit position combinations, other considerations are needed to identify the order of interactions. In the case of $E_{\text {in }}<m_{\mathrm{e}} c^{2} / 2=255 \mathrm{keV}$, the energy of the recoil electron by Compton scattering $E_{1}$ is always lower than $E_{2}$. Thus, the identification is simple. On the other hand, when the incident energy $E_{\text {in }}$ exceeds $m_{\mathrm{e}} c^{2} / 2=255 \mathrm{keV}, E_{1}$ can be larger than $E_{2}$. From the simulation, we found that events such that the photon is scattered at CdTe and then absorbed back at $\mathrm{Si}$ ("CdTe Bottom-DSSD" events) are rare. The probability is less than $2 \%$ of that of the events of reverse order ("DSSD-CdTe Bottom" events) in the energy band from 255 to $400 \mathrm{keV}$. Considering this result, we assume that a hit at DSSD is a Compton scattering when one hit is at "DSSD" and the other is at "CdTe Bottom". If two hits take place at the same detector type, "DSSD-DSSD" or "CdTe Bottom-CdTe Bottom" events, we calculate the differences between the measured $E_{1}$ and the calculated $E_{1}$ 

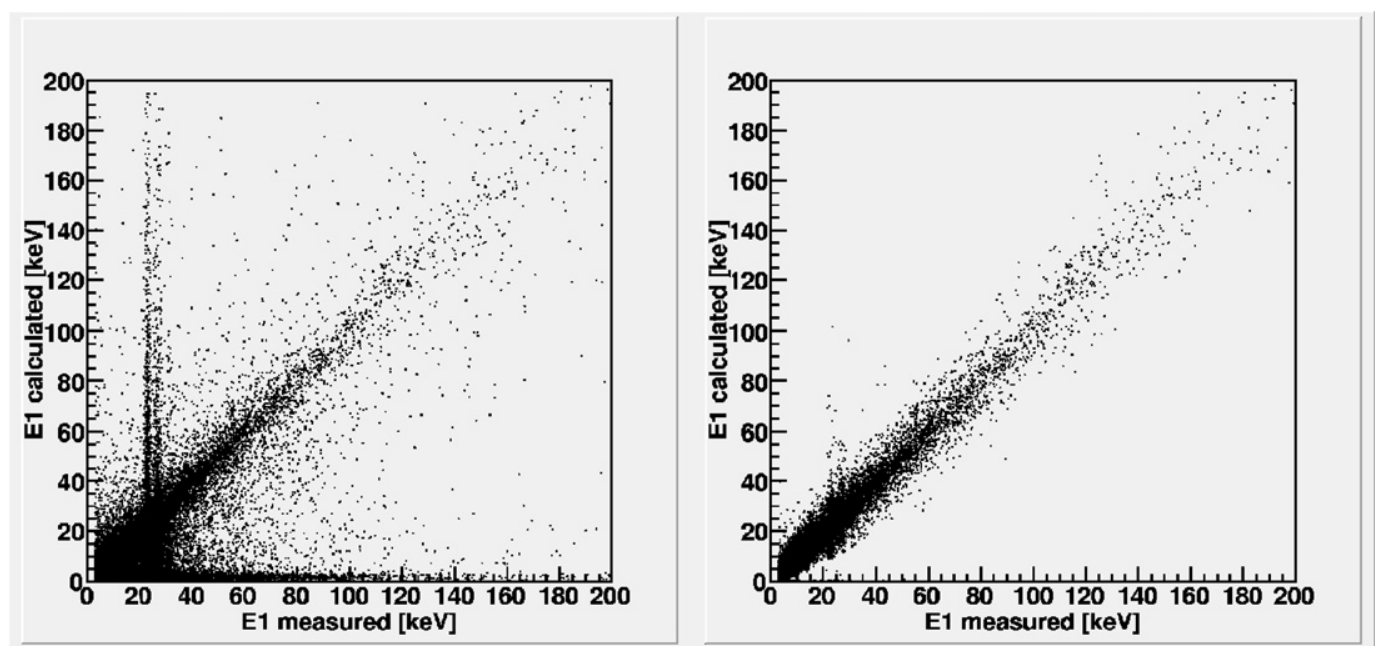

Fig. 3. Comparison of $E_{1}$ calculated by Compton reconstruction and actually measured value. The left panel stands for events after the two-hit selection, while the right panel after the "real Compton" selection.

for the two possible orders. We then select the interaction order such that the difference is smaller. By this method, we can determine the "scattering-absorption" order with an accuracy of $99 \%$ and $95 \%$ for "DSSD-DSSD" and "CdTe Bottom-CdTe Bottom" events, respectively.

Fig. 3 shows the relation between the measured $E_{1}$ and the calculated $E_{1}$ after the two-hit selection (left) and after the "real Compton" selection (right). In the case of real Compton events, the calculated $E_{1}$ is equal to the measured $E_{1}$ within the errors. The uncertainty of energy and position measured with the DSSDs and the CdTe pixel detectors contributes to the errors, as does Doppler broadening. After the two-hit selection, there are some bad events such that the calculated $E_{1}$ disagrees with the measured $E_{1}$. In these events, two hits caused by a fluorescence X-ray emitted from $\mathrm{Cd}$ or Te after photo absorption are dominant, being responsible for about $80 \%$ of the total of such events. In the left panel of Fig. 3, such events are seen as vertical lines around $20-30 \mathrm{keV}$. Other sources of error are multiple Compton scatterings or escaped electrons off the detector after photo absorption. Most of the bad events are removed after the "real Compton" selection as shown in the right panel of Fig. 3. For example, more than $90 \%$ of the fluorescence X-ray events are rejected by this selection.

\section{Detection efficiency}

The detection efficiencies in the two different data analysis modes are presented in Fig. 4. For simplicity we hereafter separate the energy band into six bands, 50-70, $70-100,100-140,140-200,200-280$, and $280-400 \mathrm{keV}$. The efficiency of the Compton mode exceeds $10 \%$ in the three energy bands below $\sim 120 \mathrm{keV}$.

Since the Compton camera is composed of DSSDs, CdTe pixel detectors at the bottom (CdTe Bottom), and CdTe pixel detectors at the sides (CdTe Side), we can classify Compton-reconstructed events into six

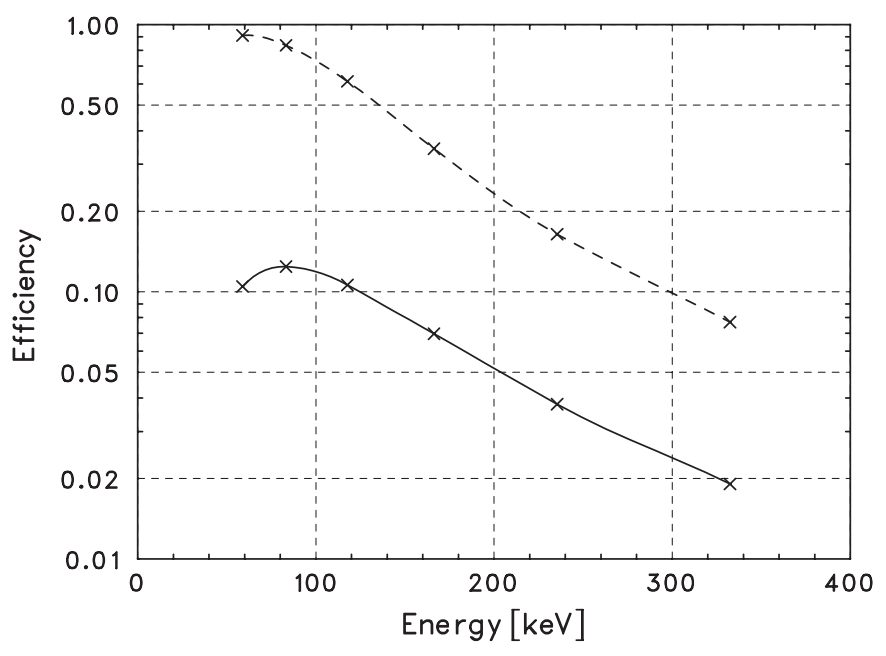

Fig. 4. Detection efficiency of the photo absorption mode (dashed line) and the Compton mode (solid line).

patterns according to their interaction positions, i.e. their detector type. Fig. 5 shows the relative ratio of hit positions of the "scatterring-absorption" events in various energy ranges. The fraction of "DSSD-CdTe Side" events are dominant in all energy bands. Below $100 \mathrm{keV}$, the probability of "DSSD-DSSD" events is the second highest. As the energy increases, the probability of "DSSDCdTe Bottom" events or "CdTe Bottom-CdTe Bottom" events increases.

Using the simulations, we evaluated what parameters of the detector design affect the detection efficiency significantly. Fig. 6 shows the detection efficiency as a function of the incident energy for different total thickness of CdTe Bottom detectors. In the default configuration, the total thickness is $2 \mathrm{~mm}$ using four layers of CdTe detectors. A thickness of $0.5 \mathrm{~mm}$ is sufficient to stop photons below $70 \mathrm{keV}$. Above $100 \mathrm{keV}$, setting the total thickness of the CdTe Bottom to $1 \mathrm{~mm}$ or greater increases the detection efficiency by a factor of 1.3-1.5. 


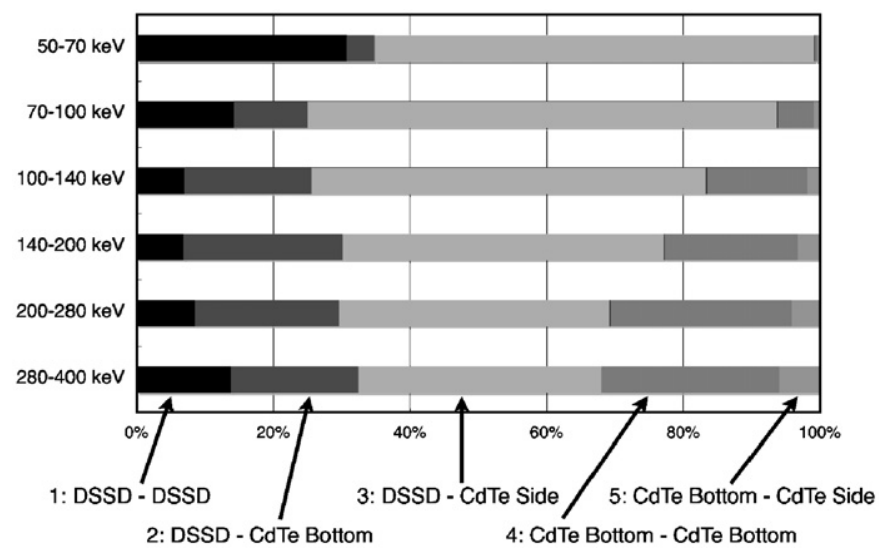

Fig. 5. Relative ratio of hit positions in the six energy bands. 1: "DSSD-DSSD" events, 2: "DSSD-CdTe Bottom" events, 3: "DSSDCdTe Side" events, 4: "CdTe Bottom-CdTe Bottom" events, and 5: "CdTe Bottom-CdTe Side" events are shown in this order from the left to the right. "CdTe Bottom - DSSD" events are so rare that they cannot be seen in this figure.

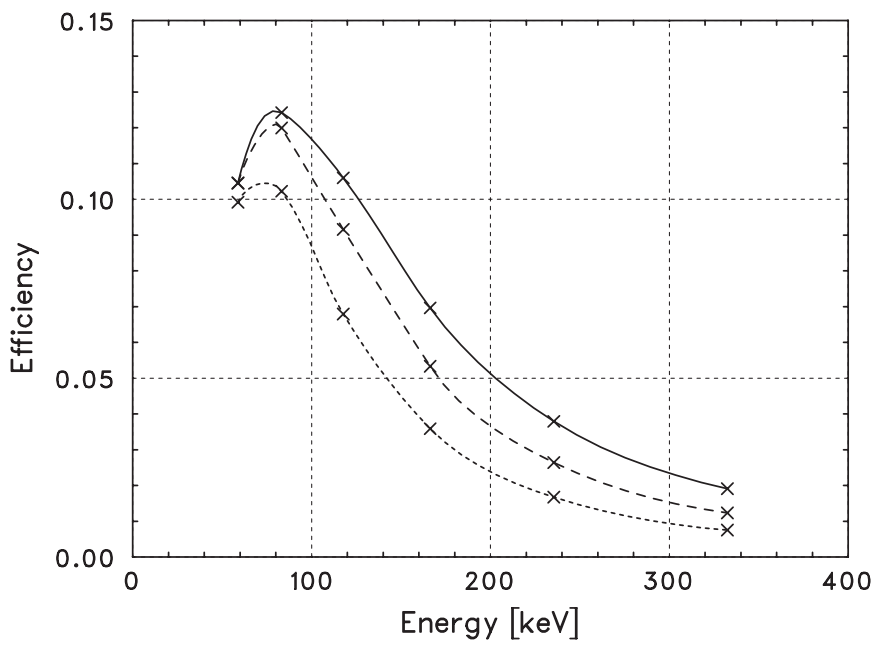

Fig. 6. Detection efficiency in Compton mode with different numbers of activated CdTe Bottom layers; results for four layers (solid), two layers (dashed) and one layer (dotted) are presented. They are equivalent to thickness of $2.0,1.0$, and $0.5 \mathrm{~mm}$, respectively.

Fig. 7 is similar to Fig. 6, but for different total thicknesses of the CdTe Side detectors. The default value is $0.5 \mathrm{~mm}$, which is equivalent to one layer of the $\mathrm{CdTe}$ pixel detector employed at the bottom layers. The detection efficiency gets much better as the thickness increases. The efficiency with $1.5 \mathrm{~mm}$ thickness exceeds $15 \%$ at around $100 \mathrm{keV}$. Since producing a high resolution CdTe detector thicker than $0.75 \mathrm{~mm}$ is technically difficult, increasing the number of layers will be a choice, even though the total number of channels will increase significantly. Adding more CdTe layers at the side improves the detection efficiency better than adding to the bottom for gamma-rays below $300 \mathrm{keV}$. This result agrees with the large proportion of "DSSD-CdTe Side events" throughout the energy band in this study.

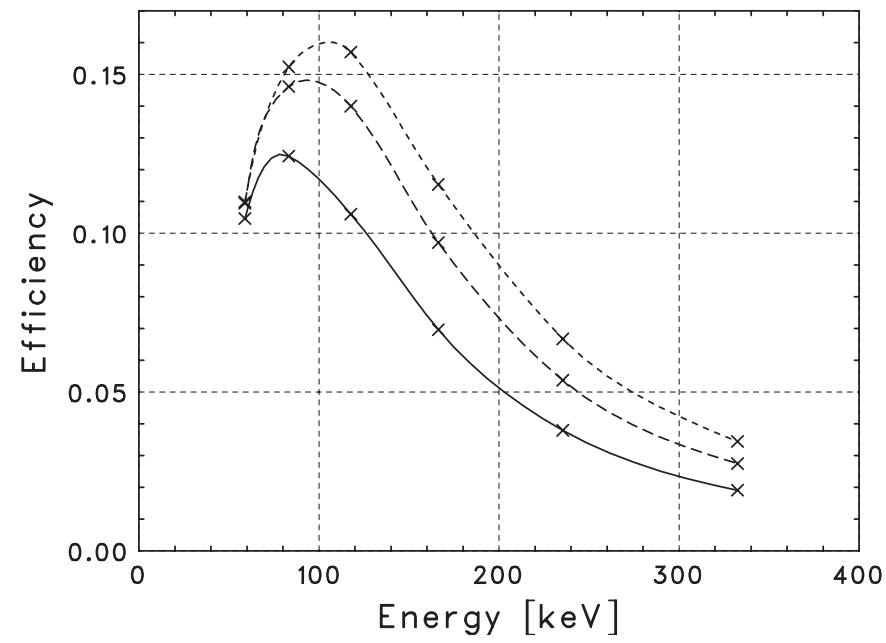

Fig. 7. Detection efficiency in Compton mode with different values of thickness of activated CdTe Side detector; results for $0.5 \mathrm{~mm}$ (solid), $1.0 \mathrm{~mm}$ (dashed), and $1.5 \mathrm{~mm}$ (dotted) are presented.

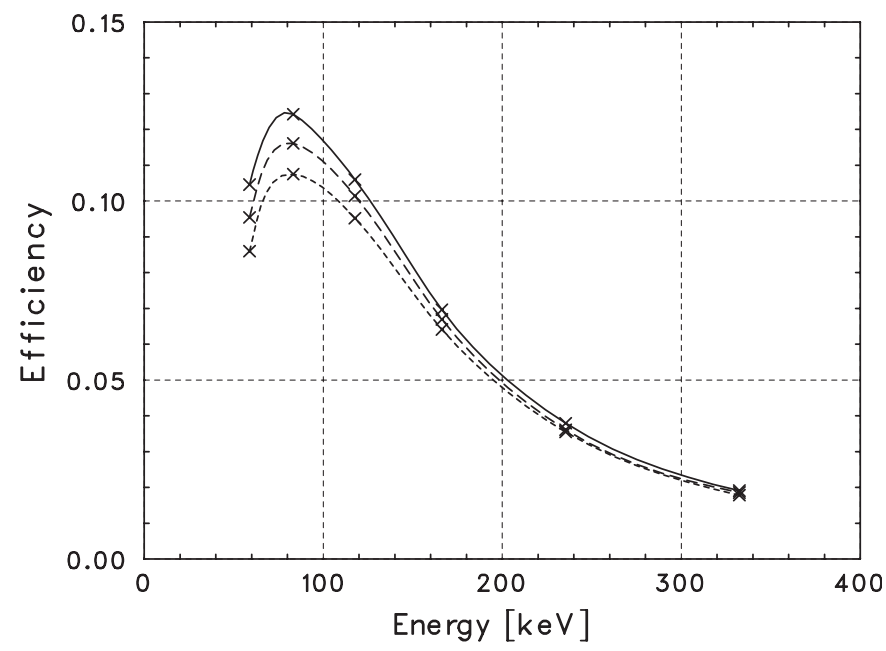

Fig. 8. Detection efficiency in Compton mode for different thickness of $\mathrm{Si}$ passive materials around detectors; results with $0.5 \mathrm{~mm}$ (solid), $2.0 \mathrm{~mm}$ (dashed), and $4.0 \mathrm{~mm}$ (dotted) are presented.

Materials surrounding the detectors, such as substrates and electronic devices for data readout, block photons and decrease the efficiency. The efficiency for several cases of the detector frame thickness is shown in Fig. 8. The material is approximated as pure $\mathrm{Si}$. The effect is not important in the high energy range above a few hundred $\mathrm{keV}$, but is significant below $100 \mathrm{keV}$.

\section{Angular resolution}

In this section we evaluate the angular resolution of the $\mathrm{Si} / \mathrm{CdTe}$ Compton telescope. We define the angular resolution measure (ARM) as $\Delta \theta=\theta_{\text {energy }}-\theta_{\text {geom }}$. Here $\theta_{\text {energy }}$ is the scatter angle calculated from energy deposits $E_{1}, E_{2}$, and $\theta_{\text {geom }}$ is that determined from hit positions geometrically. The ARM distributions in the six energy bands are shown in Fig. 9. In this figure, we use only the 

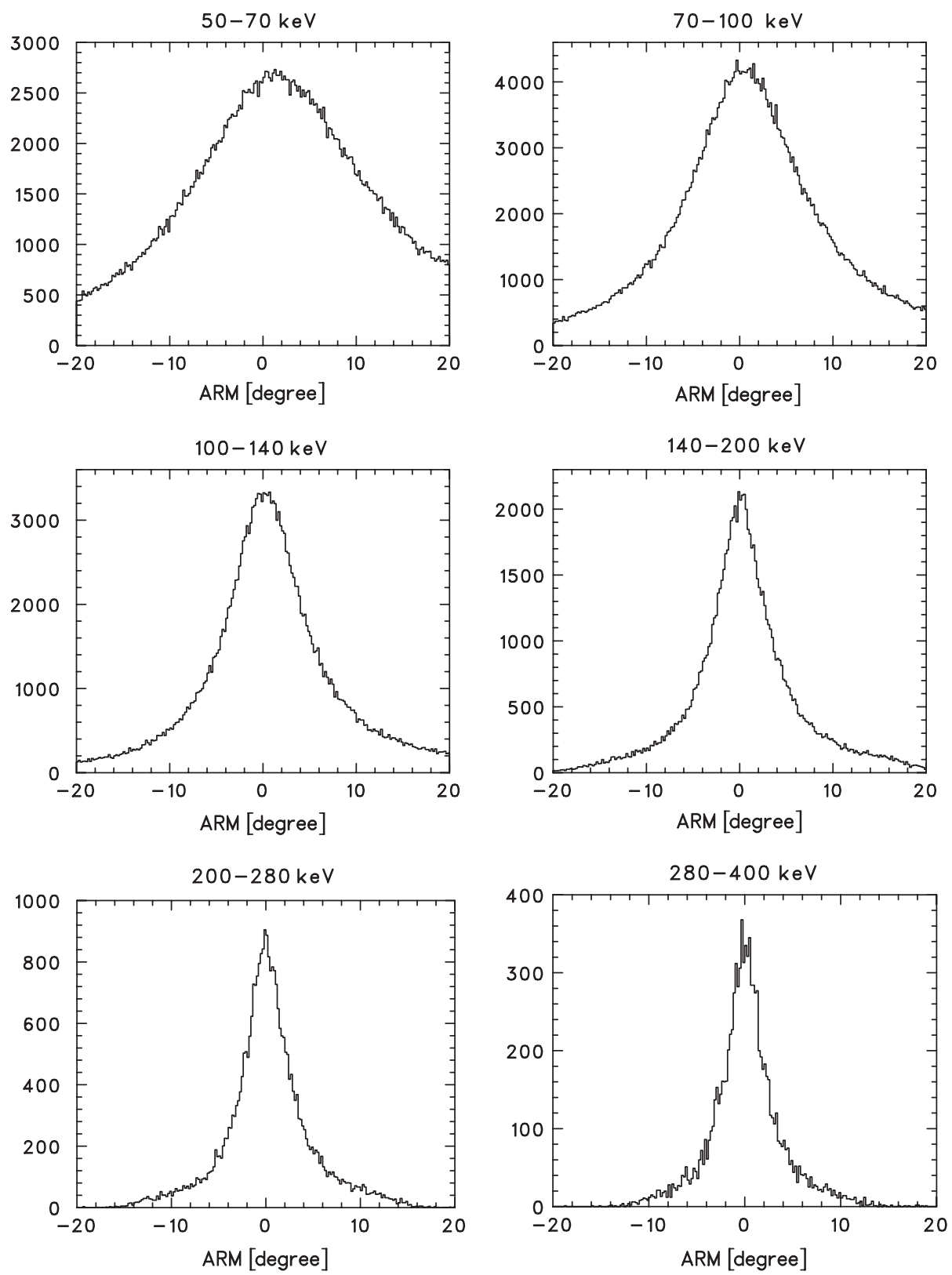

Fig. 9. Distribution of the ARM $\left(\Delta \theta=\theta_{\text {energy }}-\theta_{\text {geom }}\right)$, in the six energy bands.

"DSSD-CdTe Bottom" and the "DSSD-CdTe Side" events. As noted later in this section, the CdTe scattered events significantly deteriorate the angular resolution due to its large Doppler broadening.

The FWHM of the ARM distribution is one indicator of the angular resolution. The thick solid line in Fig. 10 shows the FWHM of the ARM distribution, $\Delta \theta_{\text {total }}$, as a function of the incident energy. As the energy increases, the angular resolution becomes better. It amounts to $9^{\circ}$ at $120 \mathrm{keV}$, and $4.4^{\circ}$ at $330 \mathrm{keV}$.

The distribution of the ARM originates from three main components: the position resolution, the energy resolution, and the Doppler broadening. Using the case of the energy band from 140 to $200 \mathrm{keV}$, we present every contribution separately by calculating the ARMs including each effect in
Fig. 11. The distributions of scatter caused by the position resolution $\left(\Delta \theta_{\text {pos }}\right)$ and that by the energy resolution $\left(\Delta \theta_{\text {ene }}\right)$ are Gaussian-like. In contrast, that of the Doppler broadening effect $\left(\Delta \theta_{\mathrm{DB}}\right)$ has a narrow peak with broad nonGaussian wings. Because the effect of these wings is significant when the three components are convoluted, the FWHM of the $\Delta \theta_{\mathrm{DB}}$ distribution underestimates the contribution of the Doppler broadening effect. In order to avoid this problem, we redefined the contribution from the Doppler broadening effect as

$\Delta \theta_{\mathrm{DB}}=\sqrt{\left(\Delta \theta_{\mathrm{total}}\right)^{2}-\left(\Delta \theta_{\mathrm{pos}}\right)^{2}-\left(\Delta \theta_{\mathrm{ene}}\right)^{2}}$.

Please note that the redefined $\Delta \theta_{\mathrm{DB}}$ is not independent of the position and energy resolutions of the detectors. 


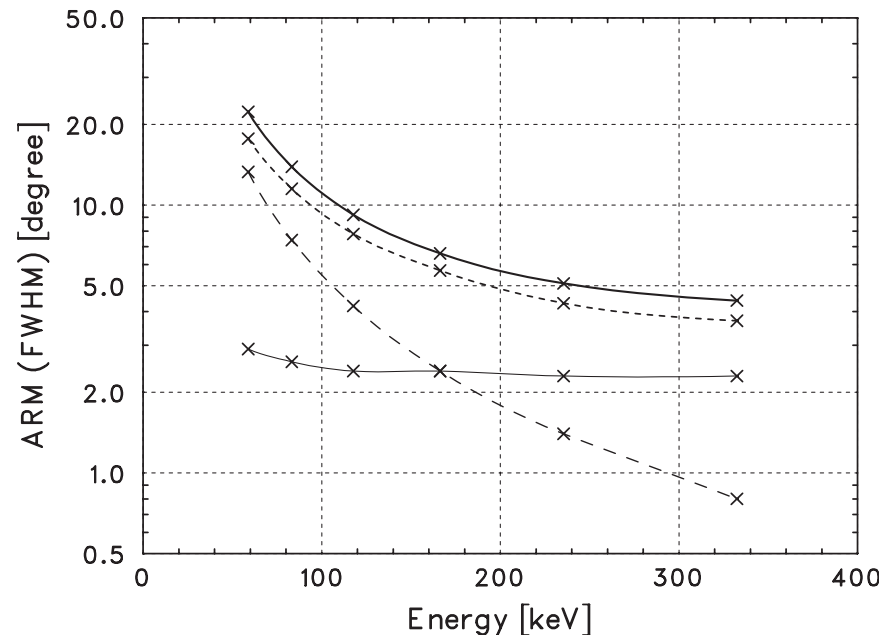

Fig. 10. FWHM of the ARM ( $\Delta \theta_{\text {tot }}$ : thick solid line) as a function of the incident photon energy. Contributions by the energy resolution $\left(\Delta \theta_{\text {ene }}\right.$ : dashed), by the position resolution ( $\Delta \theta_{\text {pos }}$ : thin solid line), and by the Doppler broadening effect $\left(\Delta \theta_{\mathrm{DB}}\right.$ : dotted line) are also presented. See text for detail.

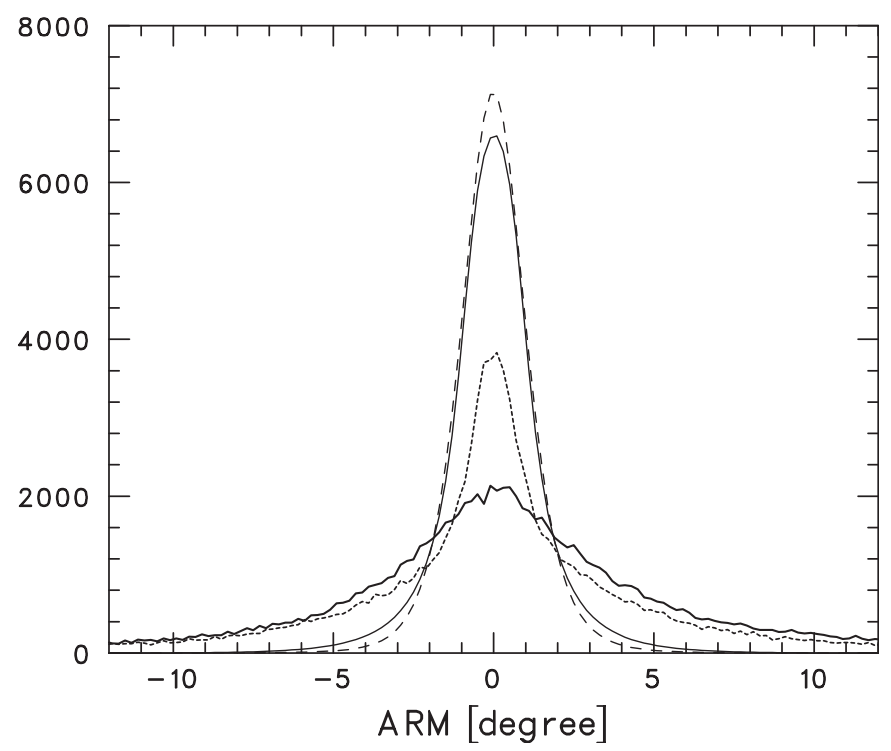

Fig. 11. The ARM distribution profile in the energy band from 140 to $200 \mathrm{keV}$ (thick solid line). Contributions by the energy resolution $\left(\Delta \theta_{\text {ene }}\right.$ : dashed line), by the position resolution ( $\Delta \theta_{\text {pos }}$ : thin solid line), and by the Doppler broadening effect $\left(\Delta \theta_{\mathrm{DB}}\right.$ : dotted line) are also presented.

The three components thus defined as a function of the incident energy are superposed in Fig. 10. The contribution from the Doppler broadening is a major component in all energy bands. Below $100 \mathrm{keV}$, the energy resolution limits the angular resolution. In the energy range higher than $200 \mathrm{keV}$, the angular resolution is limited mainly by the position resolution of the detector. If we are able to prepare CdTe pixel detectors with the finer pixel pitch of $400 \mu \mathrm{m}$, the same as that of the DSSDs, the FWHM of the ARM distribution is greatly improved from $4.4^{\circ}$ to $2.6^{\circ}$ at $330 \mathrm{keV}$. Note that this significant improvement is achieved

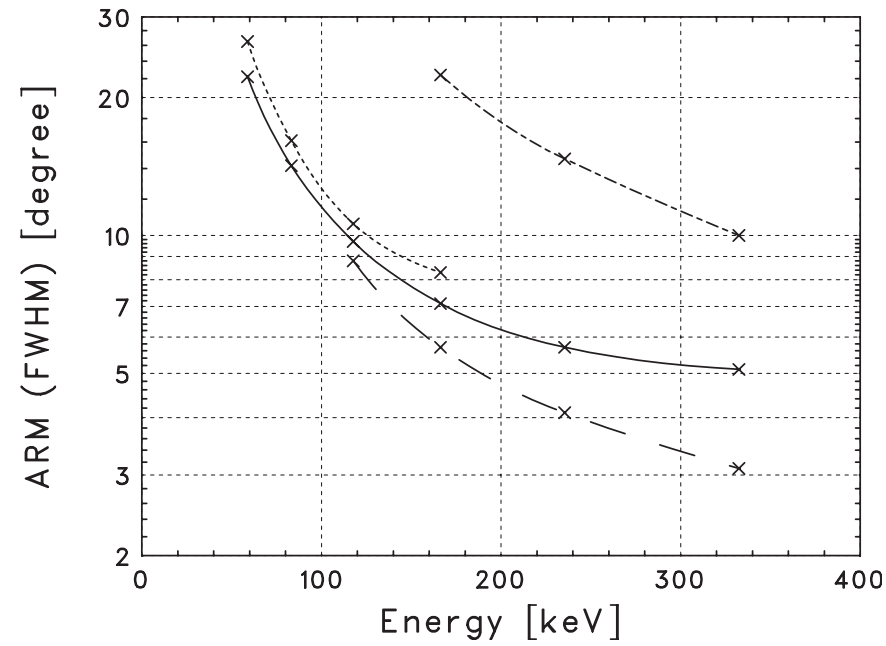

Fig. 12. The ARM of various hit positions: "DSSD-DSSD" events (dotted), "DSSD-CdTe Bottom" events (dashed), "DSSD-CdTe Side" events (solid), and "CdTe Bottom-CdTe Bottom" events (dotdash) are presented.

because the position resolution becomes smaller than the core of the Doppler broadening.

Fig. 12 shows the angular resolution for the various event types classified by hit positions (described in Section 4). We find that "DSSD-CdTe Bottom" events give the best angular resolution. At $330 \mathrm{keV}, \mathrm{FWHM}$ of the ARM with these events is $3.1^{\circ}$. In contrast, since the Doppler broadening of $\mathrm{CdTe}$ is large, CdTe detectors are not suitable as scatterers for high angular resolution Compton cameras. For example, the ARM of "CdTe Bottom-CdTe Bottom" events is significantly worse when compared to the DSSD-scattered events.

\section{Conclusion}

We carried out Monte Carlo simulations to evaluate the performances of the proposed narrow-FOV $\mathrm{Si} / \mathrm{CdTe}$ Compton telescope, the SGD, for the NeXT mission. The detection efficiency in the Compton mode at $\sim 100 \mathrm{keV}$ is more than $10 \%$. It is shown that the CdTe Side detectors play an important role in achieving better detection efficiency at $\sim 100 \mathrm{keV}$. A few degrees of the angular resolution is obtained due to the small Doppler broadening effect of $\mathrm{Si}$, combined with the high energy and position resolution of both the DSSDs and CdTe pixel detectors. At $330 \mathrm{keV}$, FWHM of ARM is $4.4 \mathrm{keV}$ and it is improved to $3.1^{\circ}$ if "DSSD-CdTe Bottom" events are selected. This result is the same level of the proposed Advanced Compton Telescope, which has an angular resolution of $3.3^{\circ}$ at $\sim 300 \mathrm{keV}$ [22]. Since our detector is optimized for a lower energy band compared with other Compton telescopes, Compton reconstruction works with the $\mathrm{Si} / \mathrm{CdTe}$ Compton telescope at lower energy such as $\sim 80 \mathrm{keV}$. For example, an angular resolution of $9^{\circ}$ is obtained for gamma-rays with an energy of $120 \mathrm{keV}$, which is very low for a Compton 
telescope. For the next step, studies of activation backgrounds caused by cosmic rays in orbit and the evaluation of the capability to reject such backgrounds is indispensable to determine detailed parameters of the detector. A Geant4 based Monte Carlo program with a capability to handle activation in the material is now being set up.

\section{References}

[1] V. Schönfelder, et al., ApJ Suppl. 86 (1993) 657.

[2] V. Schönfelder, et al., Astron. Astrophys. Suppl. 120 (1996) 13.

[3] V. Schönfelder, et al., Astron. Astrophys. Suppl. 143 (2000) 145.

[4] V. Schönfelder, New Astron. Rev. 48 (2004) 193.

[5] T. Kamae, R. Enomoto, N. Harada, Nucl. Instr. and Meth. A 260 (1987) 254.

[6] Y.F. Du, Z. He, G.F. Knoll, D.K. Wehe, W. Li, Nucl. Instr. and Meth. A 457 (2001) 203.
[7] J.D. Kurfess, W.N. Johnson, R.A. Kroeger, B.F. Phlips, E.A. Wulf, Nucl. Instr. and Meth. A 505 (2003) 256.

[8] T.J. O'Neill, D. Bhattacharya, M. Polsen, A.D. Zych, J. Samimi, A. Akyuz, IEEE Trans. Nucl. Sci. NS-50 (2003) 251.

[9] T. Takahashi, K. Nakazawa, T. Kamae, H. Tajima, Y. Fukazawa, M. Nomachi, M. Kokubun, Proc. SPIE 4851 (2003) 1228.

[10] G. Kanbach, et al., New Astron. Rev. 48 (2004) 275.

[11] T. Tanaka, et al., Proc. SPIE 5501 (2004) 229.

[12] S. Watanabe, et al., IEEE Trans. Nucl. Sci. NS-52 (2005) 2045.

[13] T. Tanaka, et al., Nucl. Instr. and Meth. A 568 (2006) 375.

[14] T. Takahashi, K. Mitsuda, H. Kunieda, Proc. SPIE 6266 (2006) 62660D.

[15] H. Tajima, et al., IEEE Trans. Nucl. Sci. NS-52 (2005) 2749.

[16] T. Takahashi, et al., Proc. SPIE 5488 (2004) 549.

[17] S. Takeda, et al., Nucl. Instr. and Meth. A (2007).

[18] Geant4, 〈http://geant4.web.cern.ch/geant4//.

[19] G4LECS, 〈http://public.lanl.gov/mkippen/actsim/g4lecs/〉.

[20] R.M. Kippen, New Astron. Rev. 48 (2004) 221.

[21] A. Zoglauer, G. Kanbach, Proc. SPIE 4851 (2003) 1302.

[22] S.E. Boggs, et al., The Advanced Compton Telescope Mission, astro$\mathrm{ph} / 0608532$, (2006). 